\title{
ROLE OF CONTRAST ENHANCED DIGITAL MAMMOGRAPHY IN BREAST LESIONS
}

\author{
Rabab Abdelmoniem, Fatma S.E Mohamed, Samer M. Botros, \\ Amr M. Abd Elsamad
}

Department of Radiodiagnosis, Ain Shams University Cairo , Egypt

Corresponding :

Rabab Abdelmoniem, Mobile : 01009137723 E mail: rabab.azab@yahoo.com Received: 13/1/2020 Accepted: 12/2/2020

Online ISSN: 2735-3540

\begin{abstract}
:
Back ground: Breast cancer in women is major public health problem throughout the world, Mammography is basic breast imaging modality for early detection and diagnosis of breast cancer, mammography has its own limitations so contrast enhanced digital mammography is new advanced application using intravenous injection of contrast under development of subtraction and dual energy technique to improve detection of breast cancer.
\end{abstract}

Aim of the Work: To investigate the potential of using intravenous contrast material with full field digital mammography to facilitate the detection and characterization of lesions in the breast.

Patients and Methods: This study was conducted on 40 patients with mammographic clinical findings that warranted biopsy recruited from department of Radio-Diagnosis, Ain Shams University hospitals. A technique for demonstrating breast cancers, dualenergy contrast agent-enhanced digital subtraction mammography, was performed for the 40female patients. The technique consists of high energy and low-energy digital mammography after administration of iodinated contrast agent. Weighted subtraction of the logarithmic transform of these images is then performed to obtain an image that preferentially shows iodine.

Results: Of the 40 subjects, twenty eight of these tumors enhanced strongly, proved carcinoma, in the other 12 patients, benign tissue enhanced diffusely in two and weakly ocally in two. These results indicate that the technique is feasible and worthy of further study.

Conclusion: Contrast-enhanced digital mammography is a new breast imaging technique using full-field digital mammography in conjunction with the injection of an iodinated contrast medium. Dualenergy CEDM also allows shorter acquisition duration and does not require prolonged breast compression. This could result in better acceptance from patients and fewer technical problems, such as misregistration of subtracted images. lesion

Key words: contrast enhanced digital mammography, breast,

\section{INTRODUCTION:}

The accuracy of mammography is limited in dense breasts where surrounding fibro glandular tissue decreases the conspicuity of lesions. Even when tumors are detected, the full extent of disease may not be clearly depicted. The growth and metastatic potential of tumors can be directly linked to angiogenesis ${ }^{(1)}$. Growth beyond a few millimeters in diameter requires the formation of new blood vessels to supply the oxygen and nutrients necessary for survival ${ }^{(1)}$. Tumor angiogenesis factors stimulate formation of abnormal vessels that 
leak and shunt blood. Therefore, imaging methods with contrast medium potentially can aid in the detection and diagnosis of cancer.

Chang et $\mathrm{al}^{(2)}$ and Sibala et al ${ }^{(3)}$ showed that contrast material-enhanced computed tomography (CT) could depict cancers in dense breasts, where conventional mammography was limited because of the lack of intrinsic tissue contrast. In the mid$1980 \mathrm{~s}$, Watt et $\mathrm{al}^{(4)}$, Ackerman et $\mathrm{al}^{(5)}$, and Watt et $\mathrm{al}^{(6)}$ performed digital subtraction angiography(DSA) of the breast by using an $\mathrm{x}$-ray image intensifier system. Benign and malignant lesions were differentiated according to the strength of enhancement.

During the past decade, considerable attention has been focused on contrastenhanced magnetic resonance (MR) imaging of the breast with gadopentetatedimeglumine as a paramagnetic contrast agent. It has been demonstrated that the region of threedimension enhancement in MR imaging correlates well with histologic assessment of tumor.

\section{AIM OF THE WORK:}

The aim of our work is to assess the role and possible indications for contrast enhanced digital mammography and its impact on breast lesions.

\section{PATIENTS AND METHODS:}

Forty women scheduled for biopsy because they were suspected of having abnormalities at breast imaging underwent imaging with contrast material enhanced digital mammography. Six sequential images of the affected breast were obtained, with a contrast agent injected intravenously between the time the first and second images were obtained. Image processing included registration and logarithmic subtraction. Lesions type, size and pathological findings were correlated with the findings at contrast enhanced digital mammography.

\section{Technique:}

Typically, the contrast-enhanced digital mammographic procedure was performed in approximately $11-15$ minutes.

This included 3 minutes for placement of the intravenous catheter, 1minute for obtaining the mask image, 1 minute or more for completion of the injection, and up to 9 minutes for acquisition of post-injection images.

The breast was lightly compressed in the craniocaudal projection for the duration of the examination, with enough pressure to limit anatomic motion but not enough to significantly reduce blood flow.

The ncraniocaudal view was used because it was easier for the patient to remain motion less in this position. In cases 12-22, imaging was performed with the production system, and an initial reference or scout mammogram was obtained with normal mammographic parameters, typically 26-32 kV, and the appropriate target and filter. The purpose of obtaining this image was to provide information regarding the soft-tissue anatomy and to provide anatomic reference points for the subsequent images that were to be obtained with identical conditions of positioning and compression.

\section{Imaging processing:}

Three major steps were performed in processing the images prior to display.

These were performed on a research display workstation, which was separate from the digital mammographic system.

First, registration between the mask image and the postcontrast images was performed to align the images as closely as possible, because a slight amount of movement of the structures within the breast occurs between each image acquisition. 
This movement can produce artifacts in the subtracted images that can distract from visualization of iodine and possibly mimic regions of uptake, which can lead to a falsepositive diagnosis.

Typically, only very small shifts $(200 \mathrm{~m})$ or 300 rotations $\left(1^{\circ}-2^{\circ}\right)$ were required; however, occasionally corrections as large as $8 \mathrm{~mm}$ were necessary.

Next, a logarithmic transform was applied to the mask image and all subsequent post contrast images.

This converted the value assigned to each pixel on the image to the natural logarithm of the original value. The transformed post contrast images were then subtracted from the transformed mask image.

The purpose of the log transformation, which is a standard operation in DSA, is to remove from the subtracted image much of the dependence on the background breast thickness and the intensity of the x-ray exposure used $(19,20)$. On the resulting set of images, any uptake of iodine appeared as a white "blush" or a region with higher pixel values than those of the surrounding tissue.

\section{RESULTS:}

At contrast enhanced digital mammography, enhancement was observed eight of
10 patients with biopsy proved cancers. Morphology generally correlated with the pathologic diagnosis. The kinetics of the lesion enhancement showed similarity to that seen with gadolinium - enhanced magnetic resonance imaging but was not consistent Patients' presentations vary as follows:

- 28/40 patients were presenting with palpable lump.

- $10 / 40$ patients presented with pain and inflammatory symptoms

- 2/40 patients received neo adjuvant chemotherapy on follow up.

- Ten of the forty $(25 \%)$ breasts had multiple lesions were diagnosed pathologically as multicentric invasive duct carcinoma. We included the dominant or largest lesion in the statistical analysis.

- Eight out of forty (20 \%) were diagnosed pathologically as benign including $1 / 8$ post-operative (and radiotherapy) edematous changes, 4/8 chronic and 3/8acute inflammatory lesions.

- $\quad 32 / 40(80 \%)$ were caused by malignant lesions; $28 / 40$ primary malignant breast masses, 4/40 cases were recurrence post conservative breast surgery.

Table 1: The total numbers of benign and malignant are shown:

\begin{tabular}{|l|c|c|}
\hline Final pathologic diagnosis & $\begin{array}{c}\text { No. of } \\
\text { cases }\end{array}$ & Percentage \\
\hline Benign & 8 & $20 \%$ \\
\hline Malignant & 32 & $80 \%$ \\
\hline Total & 40 & $100 \%$ \\
\hline
\end{tabular}

Total number of benign and malignant breast lesions causing edema and the final pathologic diagnosis. 


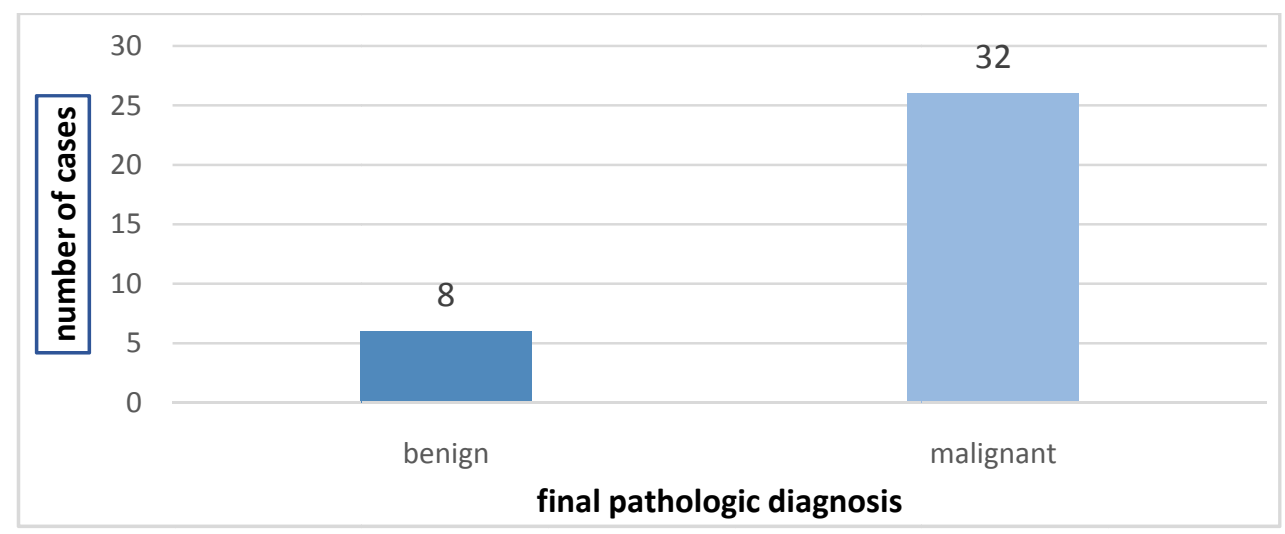

- Diagram 1: Barchart shows the total number of benign and malignant breast lesions and the final pathologic diagnosis.

- Detailed description for the number and percentage for each pathological diagnosis of breast edema causative lesion are illustrated in

Table2: Total number of cases with final pathologic diagnosis of breast edema causative lesion

\begin{tabular}{|l|c|c|}
\hline Final pathologic diagnosis & No. of cases & Percentage \\
\hline Invasive duct carcinoma grade II & 28 & $70 \%$ \\
\hline Invasive duct \& Lobular carcinoma & 4 & $10 \%$ \\
\hline Acute mastitis & 2 & $5 \%$ \\
\hline Chronic abscess & 2 & $5 \%$ \\
\hline Fibroadenoma & 4 & $10 \%$ \\
\hline
\end{tabular}

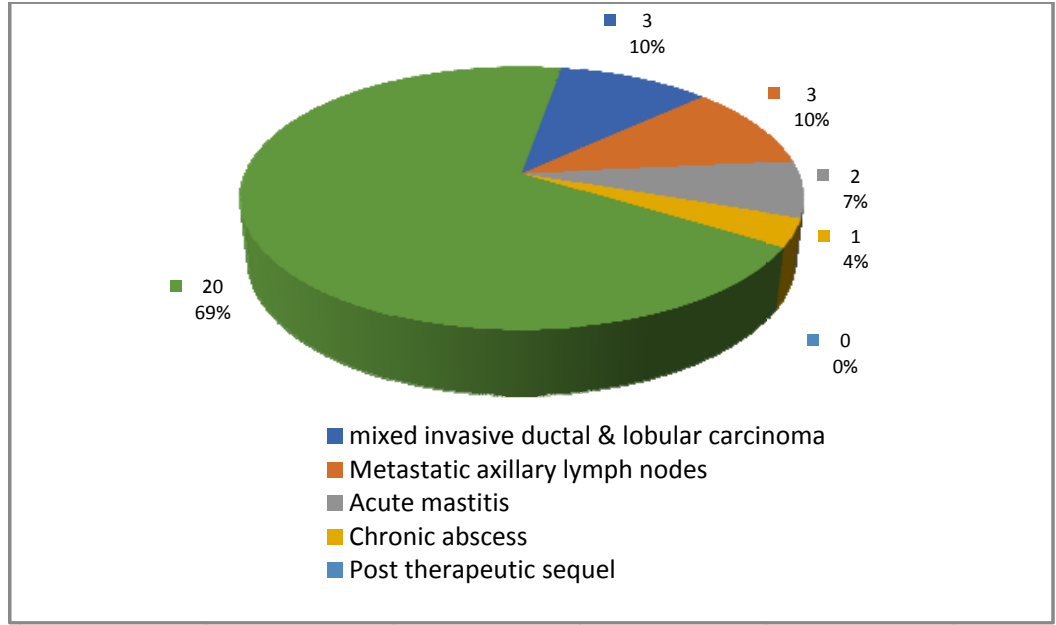

Diagram 2:Pie chart shows the final pathologic diagnosis of causative lesion 


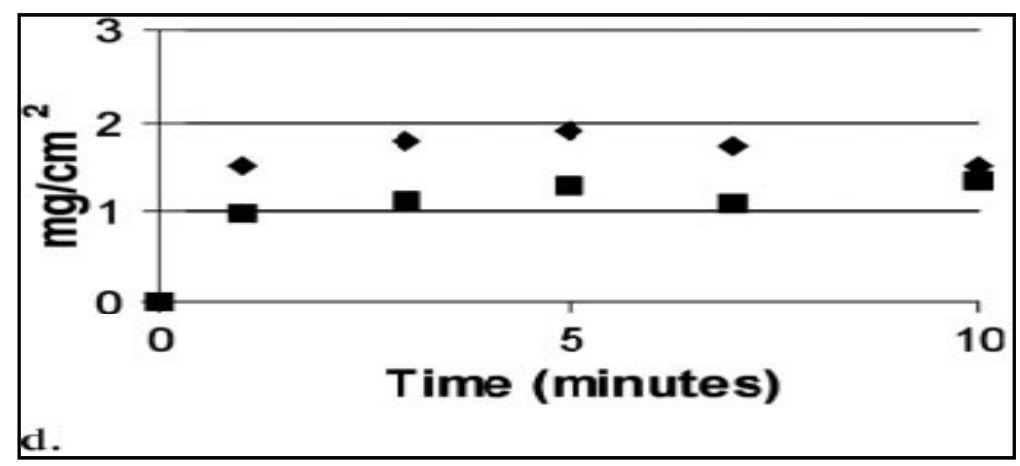

Figure 1. (a) Craniocaudal digitized screen-film mammogram obtained in a patient within filtrating ductal carcinoma (b) Craniocaudal contrast-enhanced digital mammographic subtraction image obtained 1 minute after the start of contrast medium injection shows small nodule with rim enhancement of entire mass (arrow). (c) Craniocaudal contrast-enhanced digital mammographic subtraction image obtained 10 minutes after startof contrast medium injection shows washout of contrast medium from mass. (d) Kinetic curves for the mass and an area of normal tissue adjacent to the mass. Curve for carcinoma shows early enhancement with a decrease over time, while the curve for normal tissue continues to increase at 10 minutes.

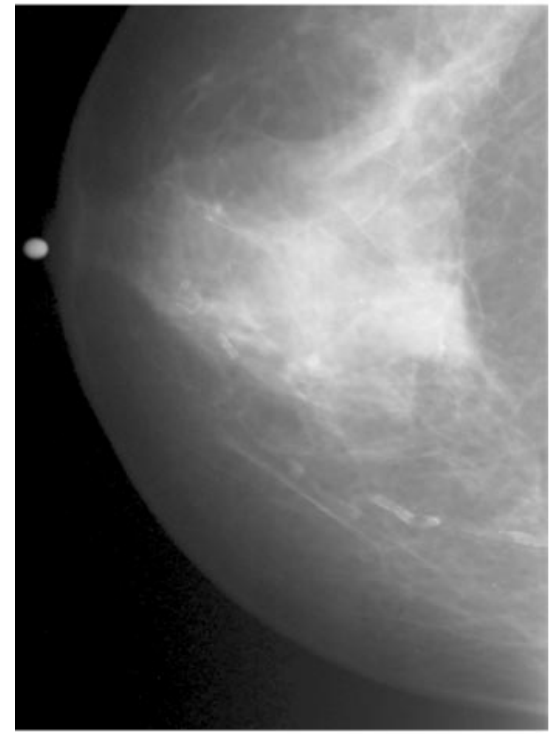

a.

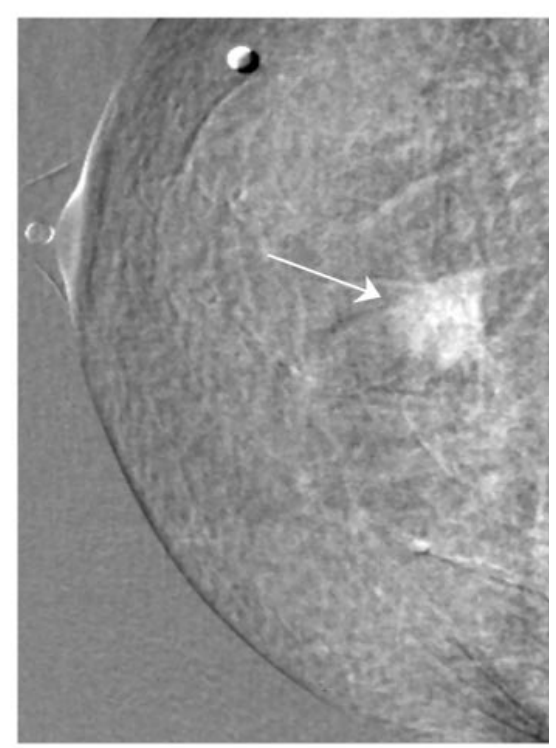

b.

Figure 2: a) Craniocaudal digitized screen-film mammogram of patient with infiltrating lobular carcinoma and ductal carcinoma in situ Metallic marker is on nipple. (b) Craniocaudal contrastenhanced digital mammographic subtraction image obtained 7 minutes after the start of contrast medium injection shows irregular spiculated enhancement (arrow). Anterior margin of mass is clearly seen on contrast-enhanced digital mammogram, whereas it is obscured by fibroglandular tissue on screen-film mammogram without contrast enhancement. Metallic marker on nipple is at left of image. Second marker overlying breast was used in early cases to aid in registration of images.

\section{DISCUSSION:}

In our pilot study, enhancement was observed in $89 \%$ (eight of nine) of the invasive cancers. There was no enhancement in seven $(58 \%)$ of the 12 benign lesions that were initially considered worrisome at mammography or US.

In the three cases with positive findings at US and negative findings at mammography and in which no 
enhancement was seen, the lesions were confirmed to be benign.

The morphology of the lesions was generally consistent with the benign and malignant features seen at other imaging modalities. However, there appeared to be less frequent enhancement in benign lesions compared with the experience at contrastenhanced MR imaging (22-26).

The kinetic curves did not consistently demonstrate distinctly different patterns for benign and malignant lesions. In some cases, however, kinetic curves were similar to those that have been reported for MR imaging.

In the presentation of the kinetics data, we have included curves for uptake, both in a region of interest corresponding to a lesion suspected of being malignant and also in an area corresponding to normal tissue. We note that in some cases on images acquired with the prototype system, the kinetic curve in the normal area appeared to assume a negative value of iodine. This was most likely caused by drifts in the output of the $\mathrm{x}$ ray tube between the mask image and postcontrast images or possibly caused by adrift in radiation sensitivity of the $\mathrm{x}$-ray detector with this prototype system.

This system is no longer operational; however, such drifts would not have been atypical with that machine. We were able to perform a simple experiment with the production system, where a uniform slab of plastic attenuator material was imaged in a sequence simulating that used in contrastenhanced digital mammography.

It was observed that in some cases the subtraction images became negative because of small drifts. However, the effect was sufficiently small (ie, typically corresponding to a projected iodine concentration less than $0.1 \mathrm{mg} / \mathrm{cm} 2$ ) to be below the threshold of detect ability of iodine.
In any case, such effects could be eliminated by including a small plastic reference object adjacent to the breast and by scaling all acquired images to hold the signal from this object constant.

The current implementation of this technique is restricted to a unilateral study of the breast, as only one breast can be monitored during the injection of contrast medium. Although a second injection is possible to image the second breast, a delay of at least 30-60 minutes would be needed between injections to minimize background enhancement in overlying tissue from the initial injection.

The use of the craniocaudal position does not allow as much breast tissue to be visualized as does the medio lateral oblique view. Because the images are projections through the entire breast, large lesions yielded stronger enhancement than did small ones, and as such, size affects quantitative interpretation of the enhancement curves. In this study, acquisition of the first image was delayed as result of our use of manual injection, which required approximately 1 minute for injection of the entire bolus of contrast medium.

However, experience from MR imaging studies of the breast suggests that a high temporal resolution is desirable to resolve early enhancement of some lesions and to provide an adequate number of temporal samples to conduct a full assessment of contrast mediakinetics.

The choice of an optimal temporal resolution depends on the way that enhancement data are to be used, and considerable variation appears in the literature.

Kuhland Schild (9) recommended that a 1 - minute temporal resolution is adequate, which is consistent with findings of this study.

However, Boetes et al (27) showed that differentiation of benign and malignant 
lesions can be improved by repetitive imaging of a lesion very 2.3 seconds. Because the volume of injected medium for breast MR imaging is smaller $(15 \mathrm{ml})$ than used in this study, it is possible that in MR imaging the tumor region may receive a more sharply defined bolus, and this could influence the measurement of lesion kinetics.

Earlier data could be obtained with this technique by administering the contrast medium more quickly with a power injector.

Now that the technique has demonstrated the ability to show cancers.

\section{Conclusion:}

The results of this preliminary study suggest that contrast enhanced digital mammography potentially may be useful in identification of lesions in the mammography dense breast. Further investigations of contrast enhanced digital mammography as a diagnostic tool for breast cancer is warranted.

\section{REFERENCES:}

1. Weidner N, Semple JP, Welch WR, Folkman J. Tumor angiogenesis and metastasis correlation in invasive breast carcinoma.N Engl J Med 1991; 324:1-7.

2. Chang J, Nesbit DE, Fisher DR, et al. Computed tomographyicmammography using a conventional body scanner. AJR Am JRoentgenol 1982; 138:553-558.

3. Sibala JL, Chang J, Lin F, Jewell WR. Computed tomographicmammography. Arch Surg 1981; 116:114-117.

4. Watt C, Ackerman LV, Shetty P, et al.Differentiation between benign and malignant disease of the breast using digital subtractionangiography of the breast.Cancer $1985 ; 56: 1287-1292$.

5. Ackerman LV, Watt $C$, Shetty $P$, et al.Breast lesions examined by digital angiography.Radiology 1985; 155:65-68.

6. Watt C, Ackerman LV, Windham JP, et al.Breast lesions: differential diagnosis using digital subtraction angiography. Radiology, 1986; 39: 159-42.

7. Holland AE, Hendrick RE, Jin H, Russ PD, Barentsz JO, Holland R. Correlation of high-resolution breast MR imaging with histopathology: validation of a technique. J Magn Reson Imaging 2000; 11: 601-606.

8. Ikeda DM, Hylton NM, Kinkel $\mathbf{K}$, et al.Development, standardization, and testingof a lexicon for reporting contrastenhanced breast magnetic resonance imaging studies. J Magn Reson Imaging; 2001; 889: 895-13.

9. Kuhl CK, Schild HH. Dynamic image interpretation of MRI of the breast. J Magn Reson Imaging 2000; 12:965-974.

10. Harms SE. Technical report of the international working group on breast MRI. J Magn Reson Imaging 1999; 10:978-1015.

11. Mussurakis S, Buckley DL, Bowsley SJ, etal. Dynamic contrast-enhanced magnetic resonance imaging of the breast combined with pharmacokinetic analysis of gadolinium-DTPA uptake in the diagnosis of local recurrence of early stage breast carcinoma. Invest Radiol 1995; 30:650662.

12. Kawashima H, Matsui O, Suzuki M, Kadoya M, Tawara M, Nonomura A, Noguchi M, Takashima T. Breast cancer in dense breast: Detection with contrast-enhanced dynamic MR imaging. Journal of Magnetic Resonance Imaging: An Official Journal of the International Society for Magnetic Resonance in Medicine. 2000; 11(3):233-43. 


\section{دور التصوير بالأشعة الرقمية للثاي بالصبغة فى تشخيص آفات الثي

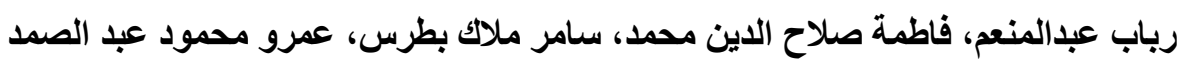

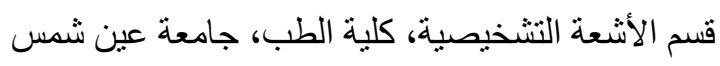

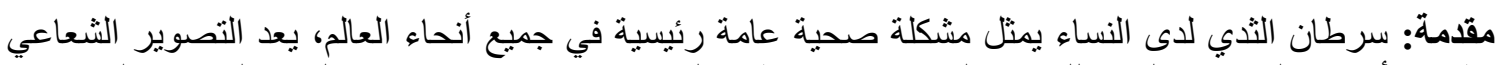

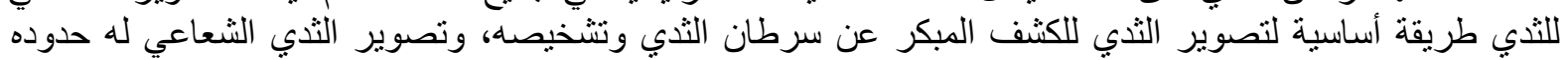

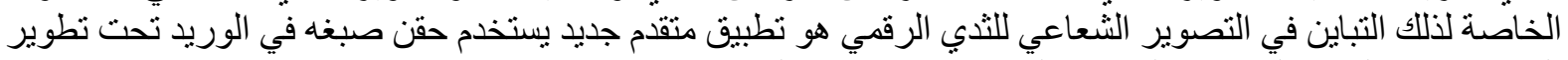
الطرح وتقنية الطاقة المزدوجة لتحسين الكثف عن سرطان الثدي.

الهدف من الاراسة: استكثاف إمكانية استخدام الصبغة في الوريد مع تصوير الثذي بالأشعة الرقمية الكامل للحقل لتسهيل اكتثاف وتوصيف الآفات في الثدي.

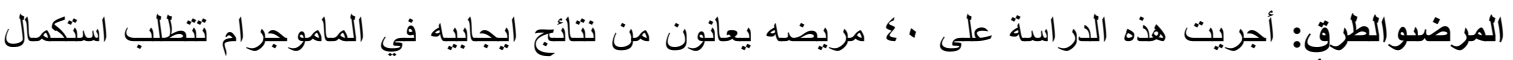

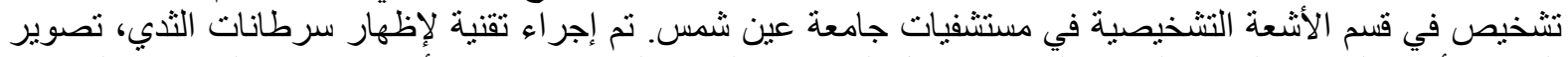

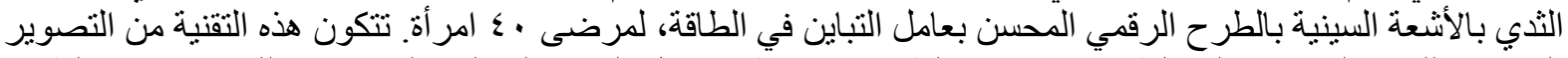

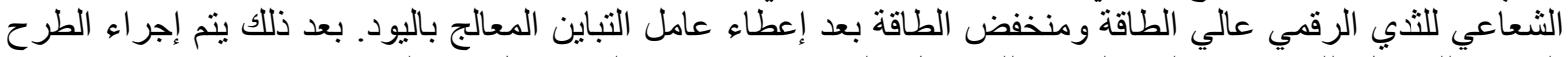

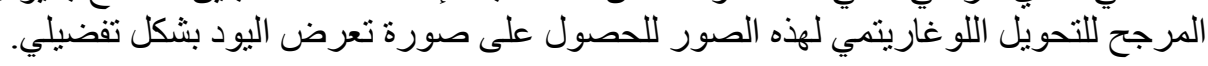

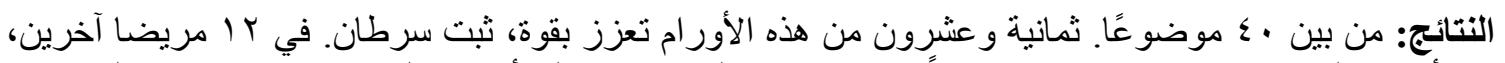

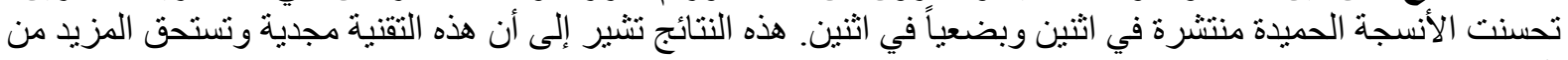

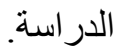

الخلاصة:التصوير الثعاعي للثدي الرقمي المحسن على النقيض من ذلك هو تلقئية جديدة لتصوير الثني تستخدم

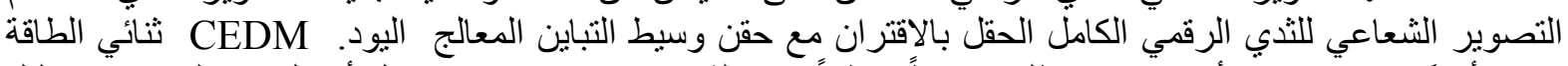

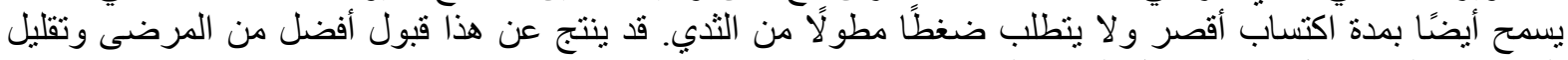
المشكلات الفنية، مثل سوء أسجيل الصنيل الصور المخصومة. 